\title{
SALVADOR TOSCANO
}

P O R
Jo s í
Ro J A s
GA R I D U E Ñ A

$I^{\prime \prime s t a n}$

NTENCIONALMENTE eludo el escribir una nota crítica o analitica de la obra de Salvador Toscano, miembro fundador de este Instituto, muerto err septiembre de 1949. Tampoco serán estas líneas un estudio bibliográfico; ello vendrá a su tiempo, han de reunirse fichas, algunos datos; seguramente eso se hará luego pero no es tal mi propósito ahora. No he investigado sistemáticamente, apenas he repasado unos cuantos papeles: artículos, estudios obsequiados con afectuosas dedicatorias de Salvador, algunas fotografias que ya principian a verse envejecidas. Por eso, más que la valoración del colega será el recuerdo del amigo lo que va en estas páginas que le dedico.

No puedo recordar, con exactitud, cuándo ni cómo conoci a Salvador Toscano; pero es indudable que ello fue en 1930, cuando él ingresó a la Escuela Nacional Preparatoria y yo a la de Jurisprudencia. Habia estudiado yo en Guanajuato el año precedente $y$, por diferencias entre los programas de allá y de aquí, faltábame pagar dos asignaturas que hube de cursar el 
mismo año en las aulas de San Ildefonso y por ahi andaba Salvador, con su grupo, ya proyectando una revista literaria que más tarde salió con el nombre de "Barandal".

Por varias circunstancias es muy probable que Salvador y yo nos hayamos conocido en la UEPOC, que por entonces canalizaba las inquietudes estudiantiles que no se conformaban con la rutina de las clases $y$ se proyectaban, acordes con la época, por la senda de las actividades sociales y políticas; en ese club o sociedad, en esa Unión de Estudiantes Pro Obrero y Campesino, acaudillados por Roberto Atwood, dando clases gratuitas a obreros jóvenes y adultos, los universitarios nos sentíamos, quién más quién menos, como una mexicana versión de aquellos estudiantes rusos que prepararon su gran revolución. Pero también pudimos conocernos en cualquier otro sitio: en aquel café improvisado en un salón del segundo patio de la "Prepa", equipado con unas extrañas mesas triangulares, de vida efímera como era de preveerse en tan desasosegado ambiente. En fin, en cualquier lugar sería y por lo mismo no se fijó en mi memoria.

En aquellos años de 1930 y 1931 no hicimos amistad íntima, pero nos veíamos con la frecuencia de quienes concurren a los mismos sitios $y$ en actividades similares. De ese tiempo surge una escena, borrosa en sus detalles pero identificable en conjunto, cuadro seguramente repetido muchas veces: de noche; en un rincón de alguno de los dos cafés de chinos próximos a la Escuela de Jurisprudencia, frente a una mesa cargada de vasos, tazas y platos, habla Alejandro Gómez Arias y lo rodea un grupo tan numeroso que las sillas se abren formando círculo, entre los que escuchamos atenta y casi devotamente veo a Toscano y a algunos otros "barandales".

"Barandal" fue revista literaria estudiantil, casi tan fugaz como otras; pero mejor que muchas de las que la precedieron y la han seguido. En la mayor parte de sus números hay colaboraciones de Salvador Toscano, que van desde un mal poema en verso libre hasta ensayos que lindan con la filosofía de la historia, pasando por un cuadro, muy al gusto de la época, que lleva por título: Stravinsky vs. Chopin (Tragedia monorrítmica en un ring). Si Salvador pudiera ver estas líneas ahora, pondria su sonrisa burlona, más de los ojos que de los labios, y comentaría con su pausada sorna: "A poco no era una vacilada muy suave."

Después escribe El sentido de la cultura en nuestro mundo, en que habla de "la desintegración definitiva de Europa" pero abriga el optimis- 
mo de una nueva civilización. El tema del momento, al menos en la Universidad de México, era Spengler y yo recuerdo que ese mismo año de 1931, en su cátedra de Historia de la Filosofia, el Maestro don Antonio Caso comentó con gran detalle y mayor entusiasmo La Decadencia de Occidente; por eso cuando en el artículo citado Toscano escribía: "Pretendo separarme fundamentalmente del pensamiento de Spengler . . .", a todos nos pareció la frase no sólo audaz sino pedante. Efectivamente lo era, pero estaba bien: un universitario de 18 años que escribe no tendrá más remedio que incurrir en ciertas pedanterías para sostener la audacia de sus mejores actitudes. En aquel articulo Toscano espera el surgimiento de un nuevo réginen, rechaza el marxismo como tesis filosófica considerándolo fórmula dernasiado simplista y rechaza también, para sí y para su grupo, igual denominación, diciendo que "se ha pretendido ver en nosotros una juventud marxista, sólo porque pretendemos el estudio de un sistema económico más justo..."; el ensayo no llega a una conclusión precisa, únicamente afirma la seguridad de que al extinguirse esta civilización, es decir el capitalismo, dará lugar a "una nueva época de cultura".

Pocos meses después rompe sus primeras lanzas en el campo de la crítica con un artículo que llamó $F u g a$ de valores; muy discutibles son las tesis que ahí sustenta, pero es interesante que afimara cosas como estas: "Tres características of rece nuestra época [es marzo de 1932] en materia de arte: una ansia de modernidad $y$ de izquierda en la juventud; una mezcla compleja de snobismo en todos los géneros artisticos, y una marcha, consciente $o$ inconsciente en el individuo, hacia un depurado clasicismo..." Adopta, él también, una posición clasicista negando la modernidad de nuestro arte porqué, dice, no hay arte nuevo ni arte viejo, hay solamente arte, que está al margen del tiempo y sobrevive a su época, "la vanguardia es una posición no un arte... comprendemos nosotros a la vanguardia como un gesto de rebeldía, pero nunca como una escuela ..." E1 snobismo es una fuerza cuyo papel es destructivo, a veces produce la extravagancia - la disolución, y concretamente censura Toscano la pintura de Mérida, la revista "Ulises" que poco tiempo antes dirigiera Novo, y Novela como Nube, de Gilberto Owen. Termina haciendo una declaración formal: "la generación literaria que nos precedió [alude indudablemente a la llamada generación de "Contemporáneos"] edificó su valer sobre las ruinas de los novecentistas, disolviendo y atacando esa generación; negando toda posible tradición; construyendo su edificio artístico sobre el sarcasmo y 
la burla. Nosottos jamás construiremos sobre ruinas, respetamos la tradición, aun la más cercana... anhelamos una obra afirmativa, con un sentido constructivo, en medio del escepticismo inteligente que nos precede."

Entre algunas aseveraciones certeras hay ahí otras muy equivocadas; los ataques concretos son extemporáneos y extraños, pues las publicaciones que menciona eran de cuatro años atrás, la novela de Owen me parece muy apreciable dentro de los lineamientos que su autor le impuso y desde luego superior a la Margarita de niebla de Torres Bodet y a otras de la misma época, y menos admisible aún el calificar de extravagancia la magnifica pintura de Carlos Mérida, independientemente del criterio que se tenga sobre el arte abstracto. Pero vuelvo a insistir sobre la fecha de aquel artículo: Toscano tenía entonces 18 o 19 años; para mí el valor de tales lineas estriba en lo que revelan de reación, asi sea un tanto arbitraria, al medio que le rodeaba. Acaso parezca exagerado, pero confieso que sigo considerando la rebeldía en el hombre como una virtud y en el joven como primordial deber.

Cuando Toscano inició su carrera de Derecho y yo continuaba la mía nos encontramos tomando parte activa en la política universitaria pero en bandos distintos. En el fondo, la inquietud y los ideales sé que eran los mismos, sólo que tratábamos de alcanzarlos por medios diferen. tes $\mathrm{y}$ en ciertos aspectos nuestras opiniones distaban mucho; pero nos sabiamos sinceros lo mismo al enfrentamos que al unimos en una empresa común, como aconteció en la huelga universitaria en octubre de 1933.

Tres años más tarde, al hacer una apreciación panorámica de -nuestra Universidad, Salvador se refirió a aquellos sucesos diciendo: "La situación de la Universidad atraviesa por su crisis definitiva los años de fronda de 1933. Para la Universidad se planteaba el problema de una filosofia adaptada a la época $y$, como punto de partida, se escogió el materialismo histórico. Los grupos que entonces lucharon contra este intento, lucharon en sentido diverso: los liberales [entre los cuales estaba Salvador] y un sector católico [en el que estuve yo], contra la tesis misma; los comunistas, contra la demagogia y subversión del orden pues, 'a un Estada marxista había de corresponder una Universidad marxista' y no precisamente a la inversa." En el mismo artículo (Trayectoria de la Unizuersidod, en la revista "Universidad", septiembre de 1936), Toscano prosigue enjuiciando la vida de la Universidad de aquella época haciendo al 
mismo tiempo una explicación y justificación del movimiento universitario de 1935 que dro la Rectoria al licenciado Luis Chico Goerne, en cuyo equipo de colaboradores figuraron, muy destacadamente, amigos íntimos de Salvador y él mismo encontró las primeras oportunidades para comenzar a realizarse en las actividades que habrian de ser su más auténtica dirección vital.

En 1936 el nuevo régimen universitario recién llegado transformó el Laboratorio de Arte, fundado un par de años atrás, en Instituto de Investigaciones Estéticas, Salvador Toscano figuró entre los fundadores y formando parte de esta institución permaneció hasta su muerte. Ya lo dijo don Manuel Toussaint mas es pertinente repetirlo aquí: el Instituto de Investigaciones Estéticas fué el centro, el medio, el ambiente que le dio apoyo y protección, propiciando en Toscano el desenvolvimiento, hasta la madurez, de una vocación y unas aptitudes que aquí mismo fructificaron; porque Salvador encontró aquí (y otros más lo hemos hallado igualmente) un suave estímulo y una ilimitada libertad de laborar, sin cortapisas de ningún género, en cualquiera dirección y en cualquiera actividad que se relacione con el estudio del arte en México.

No por iniciar sus primeras actividades como investigador había interrumpido Toscano su carrera de Leyes, por más que no sintiera verdadera atracción por las disciplinas juridicas ni por la práctica litigiosa. Terminados los estudios obtuvo la Licenciatura en 1937 presentando, como tesis para el examen de grado, un trabajo que tituló: Derecho y organización social de los aztecas; las ideas que lo guiaron en tal estudio son dos: una, el principio que llama "relativista" y que mejor convendría denominar historicista, de que "cada pueblo y momento cultural quiere su propio Derecho", y la otra consiste en considerar falsa la afirmación de que "la raíz y fuente de nuestro Derecho mexicano hay que ir a buscarla al Derecho hispano-romano", contraponiéndole la utilidad de conocer el Derecho Azteca que el autor dice no debemos tener por inoperante o muerto "pues en esta nación [México] aún teñida de elementos indígenas todavía hemos de encontrar proyectada la savia arqueológica de las instituciones juridicas aztecas", poniendo como mejor ejemplo el calpulli que sobrevivió con el nombre de ejido.

No deseo discutir esos temas, para mí tan atractivos, de la investigación y la validez de los fundamentos juridicos en que reposan nuestras instituciones; por otra parte, me consta por haber platicado expresamente sobre ello, que desde hace años Toscano consideraba que aquel trabajo 
suyo quedó con muchos puntos débiles e imprecisos $y$, posteriormente, comenzó a inclinarse por la idea de emprender una revisión total con ánimo de ampliarlo $y$ rehacerlo cuanto fuese menester. Por desgracia, creo que eso no pasó de proyecto informe, $y$ fue lástima, pues mucho habían aumentado los conocimientos de Salvador respecto a las culturas precortesianas y de seguro habria podido derivar conclusiones de mucha mayor amplitud e interés.

De cualquier manera, aquel estudio significaba, en primer lugar $y$ por la sola elección de su tema, el ya concreto y especial atractivó que en Toscano ejercía el obscuro mundo indigena de nuestro pasado $y$, después, que la preparación de tales páginas dio a su autor oportunidad y la necesidad de allegarse, seria y profundamente, a las fuentes del conocimiento histórico de las viejas culturas. Figuran en la respectiva bibliografía, códices, crónicas, obras de arqueólogos y de historiadores; con muchos de tales documentos ése sería el primer contacto, pero ya no los dejaría de mano, antes siguió frecuentando a aquéllos y a otros más y por ello, siete años más tarde, pudo Toscano darnos el fruto, ese sí maduro y pleno, en torno a otra manifestación cultural, la artística, de los pueblos precolombinos de México y Centroamérica.

Por todo eso bien podemos afirmar que de aquellos años de 1936 y 1937 arranca la actividad intelectual, ya definida y sistemática, de Salvador Toscano: artículos y notas en la revista "Universidad", donde aún alterna los temas artísticos con los sociales; notas y estudios en los "Anales del Instituto de Investigaciones Estéticas"; artículos esporádicos en diversos periódicos, en otras revistas como en "Armas y Letras" de la Universidad de Nuevo León, etc. De publicaciones aisladas recuerdo una selección y prólogo en la Biblioteca Enciclopédica Popular de la Secretaria de Educación $y$, mucho antes, una o dos pequeñas vidas de nuestros héroes que escribió para una serie de Biografías Populares, cuadernitos que durante la rectoría de Chico Goerne la Universidad imprimió, en grandes tiros, con fines de divulgación en todo el país.

E1 Instituto de Investigaciones Estéticas tuvo un paréntesis en los últimos meses de 1938, por causa de una de nuestras múltiples conmociones universitarias; reorganizado a principios de 1939, Toscano siguió laborando ahí, por entonces yo entré a formar parte del cuerpo de investigadores y la amistad con Salvador se estrechó en la convivencia de la diaria tarea. A él debo servicios que siempre agradecí mas nunca tuve ocasión de pagar: él me gestionó un curso de Historia del Arte en la Es- 
cuela Normal donde él era ya profesor; también me prestó ayuda para otra clase en la Escuela de Artes Plásticas y allí estuvimos juntos en los agitados días en que un fuerte núcleo de universitarios, profesores y alumnos, conseguimos la caída del Rector Brito Foucher, que habia implantado un régimen de tendencias y sistemas francamente facistas.

Nuestras opiniones siempre fueron discordes en lo secundario pero de acuerdo en muchos puntos primordiales; nuestras pláticas frecuentemente se convertían en discusiones pero eso no nos distanció jamás, antes la mutua amistad se afianzaba en el transcurso de los años; así entre otros amigos Salvador y yo nos acompañamos, compartiendo transportes, excursiones y alojamientos cuando hicimos algunos viajes juntos, como en aquellas memorables expediciones a Oaxaca y a Yucatán, sustentando breves cursillos de arte, bajo la guía del común amigo el licenciado Benito Coquet, entonces Director de Educación Estética.

Otros viajes hizo Toscano, siempre con fines de cultura, ya dictando conferencias como en San Antonio, Texas, en Monterrey varias veces, en Morelia y en otras partes, o en su carácter de investigador (frecuentemente combinando las dos actividades) como en sus diversas exploraciones en Yucatán, Chiapas, Guatemala y Honduras, donde recopiló observaciones, fotografías y enseñanzas de toda índole que dieron fruto en sus obras.

Así se gestó en la consulta de las fuentes, en la visita a las lejanas zonas arqueológicas, en la observación de monumentos y museos y en la depuración de la cátedra, todo el acervo de conocimientos que le permitló escribir su Arte Precolombino de México y de la América Central. Ese libro, que fue la mejor obra intelectual de Salvador Toscano, fue también, en buena parte, obra material suya, pues él proyectó, dirigió, vigiló constantemente la edición (aunque en el propio libro el colofón, por motivos burocrátiços, no mencione tal dirección del autor), que salió de las prensas upiversitarias en 1944. A su tiempo el libro fue ampliamente comentadoo, siempre con elogio y creo, al menos en lo que a mí corresponde, que esas opiniones fueron sinceras y justas. Entre lo mucho que se dijo quiero únicamente copiar aquí un párrafo que, a mi parecer, condensa un acertado juicio, el Dr. Alfonso Caso escribió: "... en Toscano se dan felizmente cita ambas tendencias, la del arqueólogo y la del crítico de arte. Su preparación en ambas ramas es indiscutible, lo que permite que los objetos arqueológicos hablen a su mente y no sólo a sus 
ojos; que sean inteligibles como objetos bellos y como productos de viejas culturas que sólo sobreviven en cuanto que son capaces de ser evocadas por nosotros".

Luego de publicar su Arte Precolombino hizo Toscano en su produc+ ción una breve pausa, pero no fue más que eso, compás de espera entre un trabajo concluído y la preparación de nuevos proyectos; conoci algunos de que me habló en diversas ocasiones y otros nos los expuso durante un almuerzo al que asistimos como invitados del Dr. Lewis Hanke, hacia mediados de 1949.

Con Pepe Porrúa celebró un amistoso convenio por el cual Salvador dirigiria una serie de publicaciones denominada Fuentes para la Historia de México, editada por la Antigua Librería Robredo, con prólogos y notas del propio Toscano. En tal forma se publicaron los dos primeros volúmenes, luego habrian de aparecer documentos como el Códice Aubin 1576, Alva Ixtlixochitl, Chimalpain, creo que también una Antología de literatura indigena mexicana. Ahora otro historiador tendrá que dirigir la serie, pero conservará el nombre de Salvador Toscano como fundador.

Otros proyectos, ya para siempre irrealizados, fueron una Historic de las Artes Populares, obra en que pensaba con gran entusiasmo; el Cuarutémoc, del que anticipó una pequeña parte en algún Suplemento Dominical del diario "Novedades"; una Antología de la poesía mexicana del siglo $X V I$, que me había pedido prologara yo. Esta era una obra proyectada hace tiempo; Salvador, que mantuvo siempre su primigenio interés por la literatura, conocía bien aquel primer siglo de nuestras letras que exploró particularmente en dos figuras: Rosas de Oquendo y Francisco de Terrazas.

Como se ve, he hablado casi exclusivamente de Toscano en sus tareas y en sus obras, es decir de Salvador Toscano como intelectual. Bien sé que este es sólo uno de sus aspectos pero, desde luego, es el que importa señalar aquí porque, lógicamente, es el que debe interesar a quienes han seguido la obra del Instituto de Investigaciones Estéticas que estos Anales reflejan. Por otra parte, yo no podría enfocarlo de modo diverso. ¿ Hablar de su carácter, de su vida intima? No me corresponde ni podría hacerlo, por varios motivos; tal vez más tarde alguien lo haga, hoy está aún demasiado cerca. Todos somos polifacéticos y presentamos a los demás perfiles diferentes; mi visión de Toscano a algunos podrá parecer débil o fallida; el tiempo dará una perspectiva mejor y plumas hay, más adecuadas que la mía, para tal propósito. 
La última vez que nos vimos fue diez o doce días antes de su muerte. Se suspendían las labores por las vacaciones en ocasión de las fiestas patrias, charlamos de literatura, de política nacional y universitaria, todo como siempre. Al final, ya despidiéndonos, lo recuerdo muy bien, cambiamos algunas ideas sobre este número 18 de nuestros Anales, entonces en proyecto; José Clemente Orozco acababa de morir, juzgamos un deber, que aquí se cumple, dedicar a su memoria algunas páginas de esta revista y Toscano of reció dar un artículo sobre algún tema, relativo a Orozco, que luego precisaría. Con tales palabras nos despedimos, deseándonos mutuamente felices vacaciones. Exactamente el día en que se reanudaron las interrumpidas labores aconteció la tragedia: el avión, perdido entre la niebla, desviado por los fuertes vientos, se estrelló en el cono inmenso del Popocatepetl; fue el lunes 26 de septiembre de 1949.

Cuando todo prometía una vida de labor fecunda, el trágico accidente suspendió la carrera y tendió el cuerpo carbonizado entre las altas rocas. Cayó a mitad del camino cuando su vida estaba en plena marcha. Había dado apenas el primer fruto de madurez, otros más se gestaban pero ya no pudieron ser. Si la cultura artística deplora tal pérdida nosotros sentimos, además, la ausencia del amigo. 


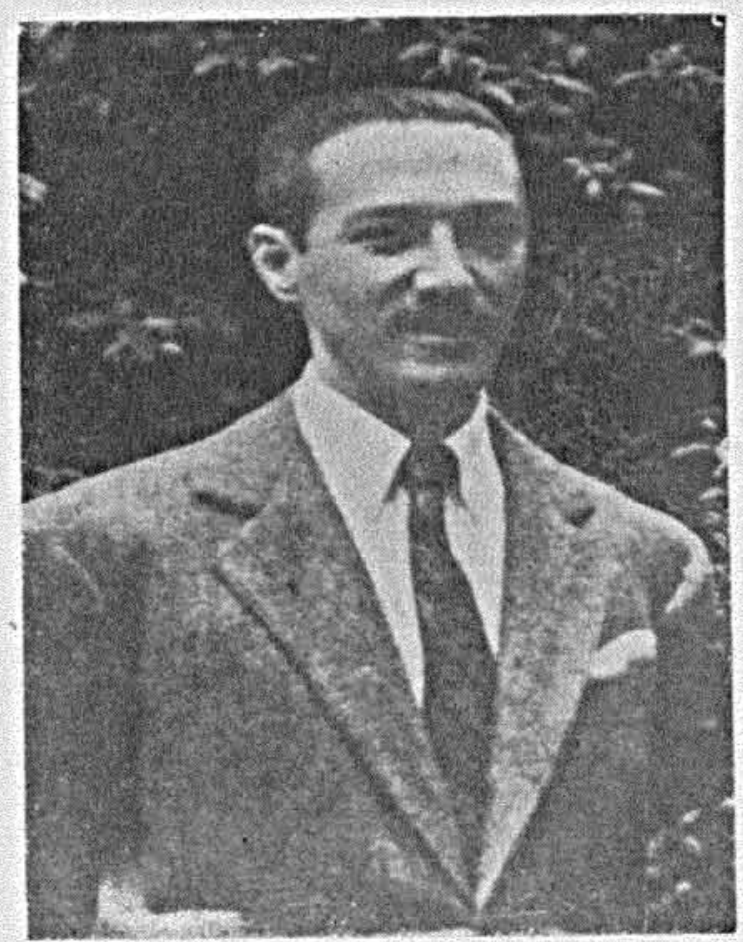

\section{SALVADOR TOSCANO}

$$
1912-1949
$$

$$
\text { F }
$$

El Instituto de Investigaciones Estéticas ha sufrido su primer desgarramiento: Salvador Toscano falleció en un accidente de aviación el 26 de septiembre de 1949 , a los treinta y seis años de edad. Lleno de vida y optimismo, dispuesto a realizar grandes empresas culturales y de investi- 
gación, he aquí que absurdamente, incomprensiblemente, ha rendido las armas. Como dijera un amigo suyo, ha muerto entre la flor y el fruto. Sus realizaciones, acogidas con general beneplácito, eran presagio seguro de obras más sazonadas: si mucho vale lo que deja ¡ cuánto no valdría lo que almacenaba en potencia! 
DOI: http://dx.doi.org/10.22201/iie.18703062e.1950.18.506

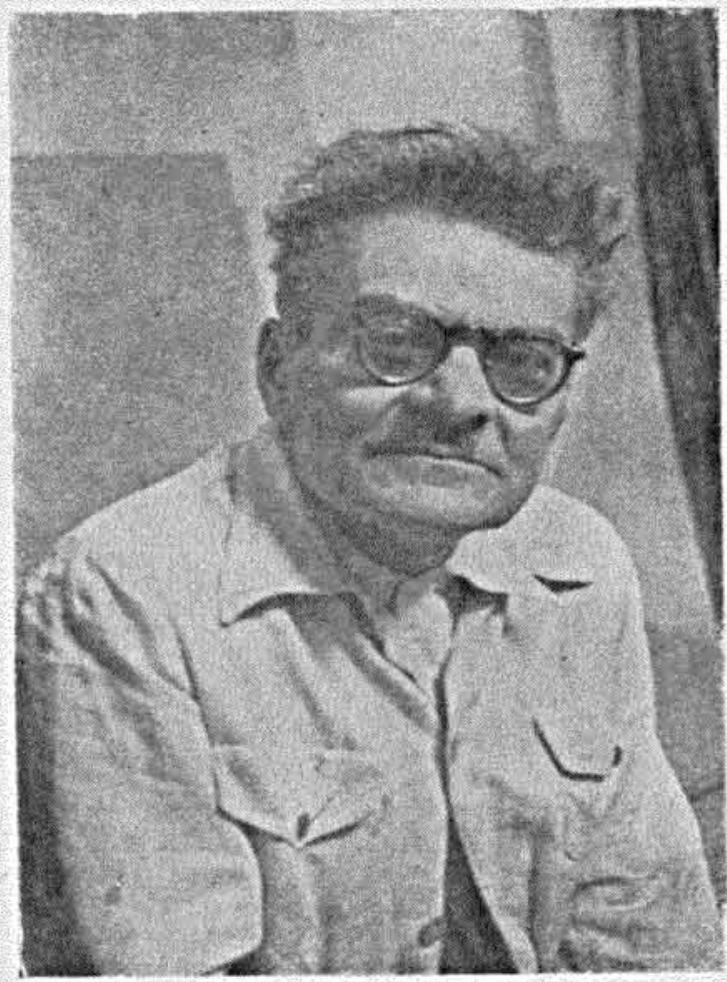

\section{JOSE CLEMENTE OROZCO}

$1883-1949$

La reciente desaparición del gran pintor mexicano José Clemente Orozco nos ha llenado de pena y, seguramente, tendrá resonancia en otros países, por tratarse de un artista cuya obra, de importancia universal, ha trascendido nuestras fronteras. Innovador original y de gran potencia 
expresiva, fué uno de los principales creadores, junto con Diego Rivera, David Alfaro Siqueiros y otros, del movimiento de pintura mural en México, que significa la máxima creación de la pintura en América. La original manera en que Orozco se expresó, la profundidad de sus concepciones, las numerosas obras de pintura mural que ejecutó en México y en los listados Unidos así como sus dibujos, grabados, litggrafías y obras de caballete, clan un carácter $y$ proporción monumentales a su vasta obra. El Instituto de Investigacione's Estéticas, que siempre siguió con atención el desarrollo del artista, desde aqui bace públicas sus condolencias por la muerte del genial pintor. 